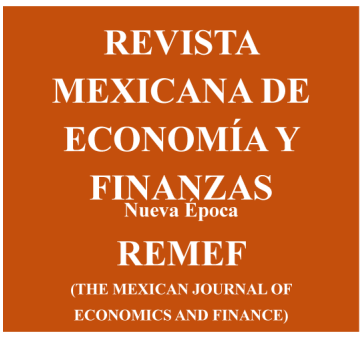
Revista Mexicana de Economía y Finanzas Nueva Época
Volumen 15 Número 3, Julio - Septiembre 2020, pp.414-434
DOI: https://doi.org/10.21919/remef .v15i3.515
(Recibido: 19/noviembre/2019, aceptado: 1/abril/2020)

fundación de investigación

\title{
Género y ahorro interno en América Latina
}

\author{
Luis Rene Caceres ${ }^{1}$ \\ University of Utah, USA
}

\section{Resumen}

El objetivo de este trabajo es cuantificar las repercusiones de la desigualdad de género en la tasa de ahorro interno de los países latinoamericanos, así como el impacto de la desigualdad de género en otras variables macroeconómicas e institucionales. Se estiman ecuaciones expresando la tasa de ahorro interno en términos de variables independientes relacionadas con la discriminación de género y con el mercado laboral. Los resultados indican que la desigualdad de género tiene un papel negativo sobre el ahorro, incrementa la vulnerabilidad macroeconómica y debilita las instituciones. Se recomienda que los países de la región emprendan grandes esfuerzos para erradicar la discriminación de género, en el marco de la ejecución de estrategias de género por país. La principal limitación del trabajo es el reducido número de datos en la muestra. La originalidad de este estudio reside en que es el primero que analiza la desigualda de genero, el ahorro y la vulnerabilidad económica en los países de América Latina. Se concluye apuntando que los países de la región deberían incrementar sus esfuerzos para lograr cerrar las brechas en este campo.

Clasificación JEL: B54, F15, F42, F43, J16, J71

Palabras claves: ahorro nacional, violencia, mercado laboral, desigualdad de género

\section{Gender and domestic savings in Latin America}

\section{Abstract}

The objective of this paper is to quantify the repercussions from gender inequality on domestic savings and macroeconomic vulnerability in the Latin American countries. Econometric equations are estimated expressing the savings rate in terms of the index of gender discrimination and of several labor market variables. Results indicate that gender inequality exerts a negative impact on domestic savings and on economic vulnerability, and weakens institutions. It is recommended that Latin American countries undertake programs designed to reduce gender inequality. The main limitation of this paper is the reduced number of data points in the sample. Its originality resides in that it is the first study that analyzes the role of gender inequality on domestic savings, macroeconomic vulnerability and institutional strength in the lstin American countries. Its main contribution is the evidence that gender inequality reduces domestic savings and increases economic vulnerability. The paper concludes pointing out the importance that the Latin American countries increase their programs designed to reduce gender inequality.

JEL Classification: B54, F15, F42, F43, J16, J71

Keywords: national savings, violence, labor market, gender inequality

\footnotetext{
${ }^{1} 5456$ Paseo General Escalón, apartamento 36, San Salvador, El Salvador, América Central. luisrenecaceres@gmail.com Sin fuente de financiamiento para el desarrollo de la investigación
} 


\section{Introducción}

En un trabajo reciente, Agenor, Ozdemir y Moreira (2018) desarrollaron un modelo de generaciones traslapadas para cuantificar las repercusiones macroeconómicas de la adopción, por un país dado, de la política pública de reducir la brecha de género en salarios. Los resultados indicaron que, entre otros efectos, las mujeres optaban por incrementar las inversiones en su educación, lo que daba lugar a aumentos subsecuentes de sus salarios y, así, adquirían mayor poder de decisión en el hogar con relación al manejo del presupuesto familiar, lo que se manifestaba en la caída del consumo corriente y en el aumento del ahorro.

Estos resultados son semejantes a los reportados por otros autores que han detectado diferencias entre mujeres y hombres en cuanto al destino de los rubros presupuestarios familiares ${ }^{2}$. Blumberg (1988), Kumar (1978), Thomas (1992) y Handa (1994) han presentado evidencia de que las mujeres son más inclinadas que los hombres a gastar los recursos bajo su responsabilidad en la nutrición de sus hijas e hijos. El análisis de Thomas (1992) de hogares urbanos de Brasil encontró que el ingreso bajo el control de la madre tenía un mayor impacto sobre la salud de la familia que el ingreso del padre. Gayer (1988) ha reportado que en Camerún las mujeres gastaban 74 por ciento de sus ingresos en la dieta familiar, mientras que los hombres gastaban solamente 22 por ciento en ese rubro. Por su parte, Kumar (1978) mostró que en Kerala, India, el nivel de nutrición de los infantes tenía correlación positiva con el ingreso de la madre, pero no respondía al ingreso del padre.

Estos estudios hacen ver que, en el seno del hogar, las mujeres tienen inclinaciones y propósitos diferentes a los de los hombres con relación al uso de los recursos monetarios bajo su control.

En el mismo sentido, también se encuentran diferencias de género en el campo de las finanzas. Dado que sus salarios son más bajos que los de los hombres y tienen responsabilidades ineludibles en el hogar, las mujeres tienden a tener mayor precaución y cautela en sus inversiones financieras. Hinz, McCarthy y Turner (1996) encontraron que las mujeres invierten en sus pensiones de jubilación de manera más conservadora que los hombres, mientras que Hungerford (1999) reportó que las mujeres contribuían mayores montos a sus planes de pensiones que los hombres. Jianakoplos y Bernasek (1998) analizaron datos del US Survey of Consumer Finances y encontraron que las mujeres solteras tenían más aversión al riesgo que los hombres solteros o casados, y que a medida de que la riqueza individual aumentaba, el porcentaje de activos riesgosos adquiridos por mujeres era mucho más bajo que el de los hombres. Por su parte, Sahay y Cihak (2018) han reportado que la participación de las mujeres en el directorio de bancos daba lugar al logro de mayor rendimiento del capital y a bajos índices de préstamos de dudosa recuperación, y que la participación de las mujeres en los entes de supervisión bancaria estaba asociada con sistemas financieros estables.

Los párrafos anteriores presentan evidencia de que cuando las mujeres aumentan sus ingresos adquieren mayor capacidad de decisión en el hogar en cuanto a la estructura del presupuesto familiar, y destinan mayores montos de recursos a rubros que benefician a sus hijas e hijos. También se desprende de la literatura reseñada que las mujeres son más adversas al riesgo financiero que los hombres.

Por otra parte, hay que traer a cuenta la evidencia de que cuando un hombre incurre en situación de desempleo, su esposa o compañera de vida entra al mercado laboral para compensar la pérdida de ingresos y mantener a flote el hogar. Este es el fenómeno del "trabajador adicional" que ha sido detectado, entre otros países, en México (Mckenzie, 2003), Argentina (Cerruti, 2000), Chile (Paz, 2008) y Brasil (Fernades y de Felicio, 2000). Así mismo, el análisis de Fuentes Castro et al. (2012) sobre las razones que inducen a las mujeres, personas con discapacidades y de la tercera edad a vender en las calles de la ciudad de México, es el propósito de contribuir ingresos al hogar. Estos casos denotan la voluntad de la mujer de proteger el hogar ante las vicisitudes económicas que se traducen en caídas del ingreso familiar.

\footnotetext{
${ }^{2}$ La reseña presentada en este párrafo se basa en Seguino y Floro (2003).
} 
Smith y Ward (1985) han reportado que la elasticidad de la participación femenina ante el aumento de los salarios es de mayor dimensión que la elasticidad de la participación de los hombres; las respectivas elasticidades son de 0.3 y -0.1 , es decir, los hombres se retiran del mercado laboral ante el aumento de los salarios, mientras que las mujeres se incorporan al mismo. Tokman (2008) ha presentado evidencia de que en Chile el aumento de la participación laboral femenina en los años noventa obedeció al aumento de los salarios.

Esta evidencia refleja el reconocimiento por parte de las mujeres de su situación endeble o precaria en el mercado laboral, ya sea porque ganan menos que los hombres, o por el acoso y las arbitrariedades que ocurren en sus puestos de trabajo, aspectos que las obliga a ser cautelosas. Es decir, además de su intrínseca o innata vocación por la protección de la familia, las mujeres son más cautelosas en materia financiera, en respuesta a la cultura de abuso y arbitrariedades en que se desenvuelven. La implicación es que, en su misión de servir de escudo al hogar, y por su aversión al riesgo ante el reconocimiento de su vulnerabilidad, las mujeres tendrán mayor propensión al ahorro que los hombres.

Seguino (2000) desarrolló un modelo postulando que el aumento del ingreso de la mujer en el hogar incrementaba su capacidad de decisión con relación al destino de los rubros del presupuesto familiar, aumentando así el ahorro del país respectivo ${ }^{3}$. Esta autora propone un modelo econométrico para explicar la tasa de ahorro interno en términos de las tasas de interés, de inflación, de dependencia demográfica, de crecimiento económico y de los términos de intercambio, incluyendo además la tasa de empleo femenino, así como la ratio de los ingresos de las mujeres a los de los hombres multiplicado por la tasa de empleo femenino. La estimación del modelo con datos panel del periodo 1975-1995 de 70 países, indico que el aumento del ingreso de las mujeres relativo al de los hombres conducía a aumentar la tasa de ahorro interno.

El papel del ahorro con relación a la inversión y al crecimiento económico ha sido objeto de un largo debate; por una parte, la posición neoclásica sostiene que el ahorro determina la inversión y por tanto el crecimiento. Por otra parte, la versión post-keynesiana mantiene que la inversión determina el ahorro y el crecimiento económico. Numerosos estudios de causalidad entre el ahorro y la inversión han demostrado para muchos países, que la causalidad se transmite, en el sentido de Granger, del ahorro a la inversión, por ejemplo, en Tunisia (Bassam, 2010), en la India (Patra y Sofi, 2016; Jangili 2011), Turquía, (Karahan, 2018), Costa de Marfil, Niger y Benin (Esso y Keho, 2010), México, (Alguacil, Cuadros y Orts, 2004), Nigeria, (Oladipo, 2010), mientras que Misztal (2010) he mostrado que la dirección de la causalidad del ahorro a la inversión ocurre tanto en países en vías de desarrollo como en los desarrollados. En base a esta evidencia, se puede inferir que el ahorro determina la inversión y el crecimiento económico y, dado que la desigualdad de género es un obstáculo a la movilización de ahorro, constituye también un obstáculo al dinamismo económico.

\section{El modelo}

En este trabajo se estima un modelo para el ahorro interno usando datos de corte transversal de 17 países latinoamericanos, correspondientes a 2016. Se reconoce que el reducido tamaño de la muestra podría dar lugar a resultados no robustos, pero ante la ausencia de mayor numero de datos los resultados deben interpretarse bajo esta limitante. Para representar la capacidad de decisión de la mujer en el hogar se usa la ratio del ingreso anual femenino al masculino, ambos per cápita, que entra en las ecuaciones como una variable independiente $\mathrm{y}$, en otros casos, entra multiplicada por la tasa de empleo femenino para representar el acceso de las mujeres al mercado laboral ${ }^{4}$.

\footnotetext{
${ }^{3}$ Véase también Seguino y Floro (2003).

${ }^{4}$ Los países incluidos en la muestra son los países latinoamericanos del continente americano, excepto Venezuela RB, y se ha incluido a la Republica Dominicana.
} 
Un primer modelo usa el indicador de desigualdad de género, (IDG), como variable independiente. Este índice comprende lo referente a la desigualdad de género con respecto a la salud (la mortalidad maternal y la tasa de fertilidad de adolescentes), la brecha en empoderamiento (las brechas en educación secundaria y de sillas en el parlamento) y la brecha en la participación en la economía (la brecha en las tasas de participación laboral). El IDG muestra coeficientes significativos y negativos, de lo que se deduce que el género impacta al ahorro no solo a través del ingreso femenino relativo al masculino, sino también por medio de la discriminación contra las mujeres en cuanto a salud, participación laboral y empoderamiento. En este modelo también se incluyen la ratio del empleo femenino al masculino, así como el porcentaje de las remesas a PIB, como variables independientes.

En un segundo modelo, se usa el índice de oportunidad humana, (IOH), desarrollado por el Banco Mundial (Molinas et al., 2011), que es un indicador de movilidad social. El IOH mide el grado en que las niñas y los niños de un país dado tengan iguales oportunidades de acceso a servicios de educación, salud, agua, electricidad, vivienda, así como también a terminar el sexto grado en el periodo reglamentario, independientemente del ingreso de sus padres y del lugar de residencia. Este índice tiene altas correlaciones con el crecimiento económico y con el dinamismo de las exportaciones, como se muestra en Cáceres (2018).

Además del IOH, este modelo usa otras variables independientes, el ratio del ingreso femenino al masculino, la tasa de dependencia demográfica, la dimensión de la economía subterránea como porcentaje del PIB, y el porcentaje de la población femenina con educación secundaria.

En este trabajo se abordan diversos temas porque la literatura sobre las implicaciones macroeconómica de la desigualdad de género no ha recibido mucha atención en América Latina. Se espera que el análisis de varios temas servirá para motivar a investigadores a desarrollar esos temas en trabajos de mayor extensión.

Este trabajo muestra resultados que no han sido reportados en la literatura sobre el papel de la desigualdad de género, específicamente su papel en incrementar la vulnerabilidad macroeconómica de un país en cuestión; los resultados que muestran el impacto de la brecha de ingresos femenino y masculino sobre la violencia intrafamiliar tampoco han sido reportados en la literatura para los países latinoamericanos. Además, este es el único trabajo que trata exclusivamente con los países latinoamericanos, y junto con el estudio de Seguino (2000), se sustenta en la estimación de ecuaciones econométricas, ya que el trabajo de Agenor et. al. es un modelo de equilibrio general.

\section{Los datos y sus características}

La fuente de datos es el Reporte del Desarrollo Humano 2016, (UNDP, 2016) excepto en casos en que se señala otra fuente de datos específica. Todas las variables fueron objeto de pruebas de raíz unitaria por medio del estadístico ADF ampliado, con resultados que indicaron que las siguientes variables eran integradas de orden uno: ahorro interno, participación masculina, participación femenina, estado de derecho, tasa de dependencia, shadow, ingreso masculino, ingreso femenino, remesas, ingreso femenino/ingreso masculino, $\mathrm{IOH}^{*}$ (ingreso masculino/ingreso femenino). Otras variables resultaron ser estacionarias al nivel de 5 por ciento: empleo femenino, empleo masculino, participación femenina/participación masculina.

En vista de que las ecuaciones a ser estimadas incluyen variables estacionarias así como otras que son I(1), se optó por realizar las estimaciones usando la metodología de "Fully Modified Least Squares" de Phillips y Hansen (1990). Se reconoce que las ecuaciones estimadas presentadas en los cuadros 1, 2 y 3 pueden ser sujetas a problemas de endogeneidad y de variables omitidas. 


\section{Resultados}

\subsection{Primer modelo}

Se observa en el gráfico 1 que existe una asociación muy estrecha entre el IDG y la tasa de ahorro interno; el valor negativo de ahorro interno corresponde a El Salvador.

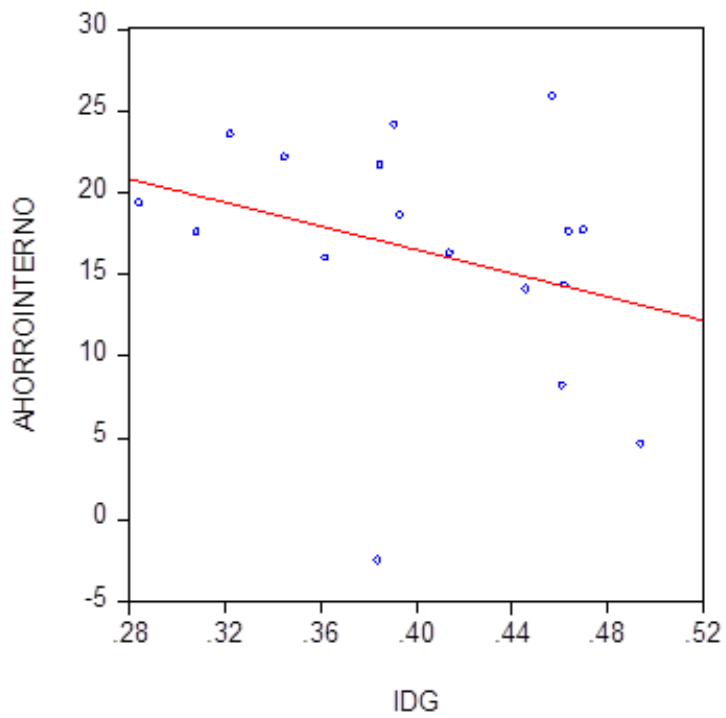

Gráfico 1. IDG y tasa de ahorro interno.

Fuente: elaboración del autor.

La ecuación (1) del cuadro 1 muestra que el IDG tiene un coeficiente negativo y significativo y, con la variable cualitativa Cualies, que representa el caso de outlier de El Salvador, explica la mitad de la varianza de la tasa de ahorro interno. Es decir, la desigualdad de género tiene un papel relevante en la determinación del ahorro interno, ya que refleja las restricciones que las mujeres enfrentan en el mercado laboral, así como en su acceso a educación y salud, y en la participación política, en el contexto en que la obtención de ingresos por parte de la mujer tiene incidencia en el ahorro. Así mismo, la desigualdad de género, en cuanto socaba la operación de los mercados, especialmente el laboral, mina la productividad y acarrea pérdidas de eficiencia que se traducen en pérdidas de ingresos y, por ende, del ahorro interno.

En la ecuación (2) la ratio de las tasa de empleo femenino a masculino tiene un coeficiente positivo y significativo, y la ecuación explica 64 por ciento de la varianza del ahorro interno. Este resultado corrobora que el aumento del empleo femenino relativo al masculino dota a la mujer de mayor capacidad de decisión en el hogar y, por tanto, el ahorro aumenta.

En la ecuación (3) se usa la variable compuesta que resulta de multiplicar el IDG por la ratio del empleo masculino al femenino, y su coeficiente es negativo y significativo. En este caso, el IDG potencia el efecto perverso sobre el ahorro de la mayor ratio del empleo masculino al femenino.

El porcentaje de la ratio de remesas a PIB, Remesas, muestra un coeficiente significativo y negativo, lo que está de acuerdo con varios estudios que han mostrado que las remesas se disipan en el consumo de bienes importados y no contribuyen al crecimiento económico (Cáceres, 2018). Otra explicación podría radicar en la evidencia de que las remesas reducen la participación laboral femenina, tal como ha sido reportado para los casos de El Salvador (Acosta, 2006), Haití (Jodette, 2009) y Filipinas (Rodríguez and Tiengson, 1991). 
Cuadro 1. Determinación del ahorro interno.

\begin{tabular}{|c|c|c|c|}
\hline \multicolumn{2}{|c|}{ VARIABLE INDEPENDIENTE: TASA AHORRO INTERNO } \\
\hline & \multicolumn{3}{|c|}{ Ecuacion numero: } \\
\hline Variable Independiente: & $(1)$ & $(2)$ & $(3)$ \\
\hline Constante & 39.623 & 13.1381 & 28.4674 \\
\hline Cualies & -23.3428 & -21.7817 & -15.4184 \\
\hline IDG & -55.4268 & & \\
\hline Empleo Femenino/ & & 30.3652 & \\
\hline Empleo Masculino & & $(2.76)$ & \\
\hline IDG (Empleo Masculino/ & & & -14.2438 \\
\hline Empleo Femenino) & & & $(2.15)$ \\
\hline Remesas & & & -0.434 \\
\hline & & & $(1.89)$ \\
\hline R2 & 0.53 & 0.64 & 0.75 \\
\hline
\end{tabular}

Fuente: elaboración del autor.

\subsection{Segundo modelo}

En la ecuación (1) del cuadro 2, la variable independiente es el índice de oportunidad humana, que tiene una asociación estrecha con el ahorro interno, como se observa en el gráfico 2. El IOH tiene un coeficiente positivo y significativo y la ecuación explica el 63 por ciento de la varianza del ahorro interno.

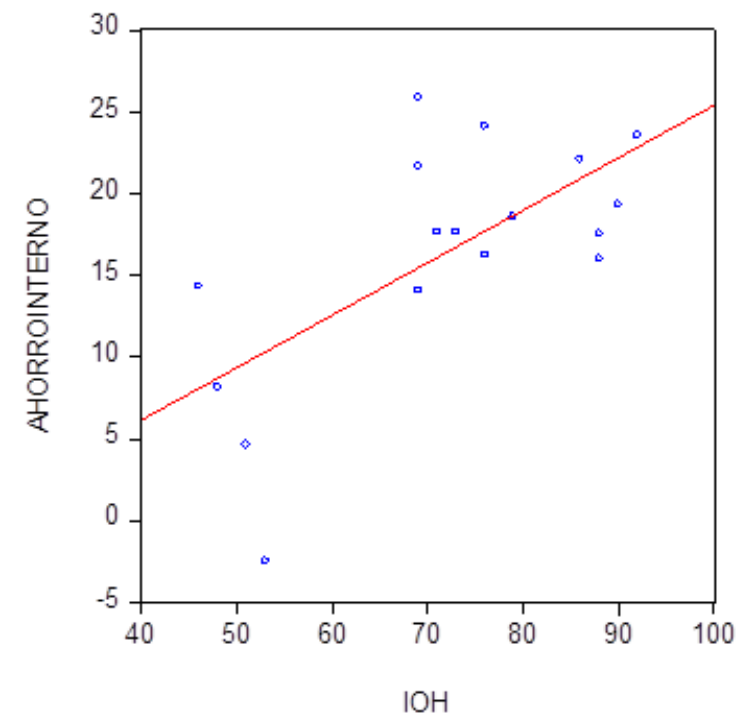

Gráfico 2. IOH y tasa de ahorro interno.

Fuente: elaboración del autor.

Este índice es una medida de las oportunidades de movilidad social existentes en un país dado. La implicación es que el ahorro interno depende del régimen de oportunidades que existe en un país, o sea de la igualdad de oportunidades de acceso a los servicios de educación, agua, electricidad, salud y otros, lo cual redunda en incrementar el capital humano y los ingresos, especialmente de las mujeres.

En la ecuación (2) se introduce como variable independiente la ratio del ingreso femenino al masculino, que muestra un coeficiente positivo y significativo y la ecuación explica 68 por ciento de la varianza.

En la ecuación (3), la variable compuesta $\mathrm{IOH}^{*}$ (ingreso femenino/ingreso masculino) entra como variable 
independiente y tiene un coeficiente positivo y significativo. La otra variable independiente es la tasa de dependencia, es decir, el porcentaje de la población menor de 15 años, la cual tiene un coeficiente negativo y significativo, como era esperado.

En la ecuación (4) se introduce una variable independiente igual a la ratio del ingreso femenino al masculino multiplicado por la tasa de empleo femenino, que tiene un coeficiente positivo y significativo, mientras que la variable Shadow, que representa la dimensión de la economía subterránea, tomada de Buehn y Scheneider (2016), tiene un coeficiente negativo y significativo. Cáceres (2017) ha demostrado que esta variable tiene una asociación positiva con la tasa de pobreza en los países latinoamericanos, y que representa un indicador del empleo precario, lo que no es propicio al ahorro.

La ecuación (5) muestra que el coeficiente del porcentaje de mujeres con algún grado de educación secundaria, Población femenina, es positivo y significativo y la ecuación explica 79 por ciento de la varianza, poniendo de relieve el papel singular de la educación de las mujeres en la movilización del ahorro.

En vista del impacto negativo de la desigualdad de género sobre el ahorro, la reducción de esta desigualdad, por ejemplo, mediante la reducción de la brecha de salarios, o la brecha de participación laboral, conduciría a aumentar el ahorro y, por tanto, la economía estaría en mejor capacidad de atender un mayor nivel de inversión. De esa manera, la reducción de la desigualdad de género es un medio para dinamizar la economía.

O sea que la desigualdad de genero debería verse como un legítimo mecanismo de política macroeconómica al que se puede recurrir para impulsar el crecimiento económico; es decir, el IDG no es exclusivamente una variable de tipo "social", pero es sobre todo un instrumento propio de la macroeconomía, y que, además, establece un vínculo entre el respeto a los derechos humanos y la macroeconomía.

Cuadro 2. Determinación de la tasa de ahorro interno.

\begin{tabular}{|c|c|c|c|c|c|}
\hline \multicolumn{6}{|c|}{ VARIABLE INDEPENDIENTE: AHORRO INTERNO } \\
\hline & \multicolumn{5}{|c|}{ Ecuacion numero: } \\
\hline Variable independiente: & $(1)$ & $(2)$ & $(3)$ & $(4)$ & (5) \\
\hline \multirow{2}{*}{ Constante } & -0.0366 & -13.6696 & 14.2077 & 11.0532 & -12.344 \\
\hline & $(0.56)$ & $(1.96)$ & $(5.40)$ & $(1.68)$ & $(2.34)$ \\
\hline \multirow{2}{*}{ Cualies } & -16.214 & -15.1023 & -17.345 & -19.745 & -15.242 \\
\hline & $(3.75)$ & $(4.45)$ & $(5.30)$ & $(4.00)$ & $(4.41)$ \\
\hline \multirow{2}{*}{$\mathrm{IOH}$} & 0.2802 & 0.2648 & & & \\
\hline & $(3.77)$ & $(4.41)$ & & & \\
\hline \multirow{2}{*}{ Ingreso feme/Ingreso masc } & & 20.59 & & & \\
\hline & & $(1.82)$ & & & \\
\hline \multirow{2}{*}{$\mathrm{IOH}$ (ingreso feme / ingreso masc) } & & & 0.3092 & & \\
\hline & & & $(1.88)$ & & \\
\hline \multirow{2}{*}{ Tasa dependencia } & & & -0.2227 & & \\
\hline & & & $(1.88)$ & & \\
\hline \multirow{2}{*}{$\begin{array}{c}\text { (Ingreso feme / ingreso masc) } \\
\text { (empleo feme) }\end{array}$} & & & & 0.653 & 0.3732 \\
\hline & & & & $(2.88)$ & $(2.40)$ \\
\hline
\end{tabular}

Fuente: elaboración del autor.

\section{5. Índice de oportunidad humana y tasa de ahorro interno}

Llama la atención que la relación entre el $\mathrm{IOH}$ y la ratio del ingreso femenino al masculino, que se observa en el gráfico 3, no es lineal sino cuadrática, y muestra rendimientos decrecientes después de que el IOH supera el valor de 0.72. Altos valores del IOH corresponden a los países con más altos niveles de PIB per cápita, 
de manera que el gráfico 3 significa que, en la medida en que el IOH aumenta, la economía se vuelve más compleja, y la disparidad entre los ingresos correspondientes a hombres y mujeres se acentúa en favor de los primeros.

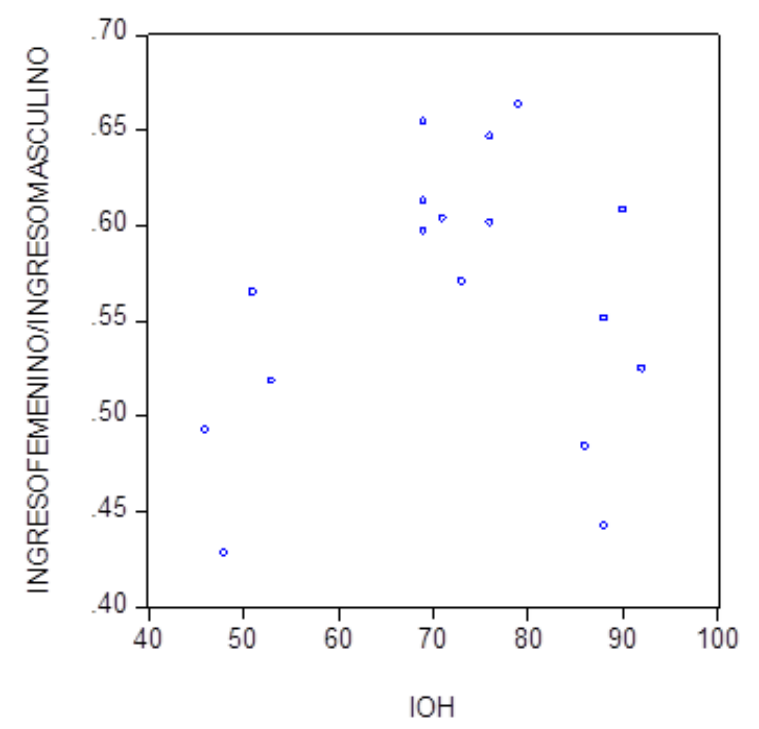

Gráfico 3. IOH y ratio de ingreso femenino a masculino.

Fuente: elaboración del autor.

La ecuación estimada es la siguiente:

Ingreso femenino/ingreso masculino $=$

$-0.6143+0.0347 \mathrm{IOH}-0.00247(\mathrm{IOH})(\mathrm{IOH})$

(2.02) (3.82) (3.73)

$\mathrm{R}$ cuadrado $=0.52$

Este resultado se puede interpretar como una manifestación de la segregación ocupacional de las mujeres quienes, aunque existan oportunidades de educación superior, continúan optando por las carreras "tradicionales" de enfermería, magisterio, secretariado, mientras que los hombres escogen carreras donde se ganan salarios altos, como las ingenierías, las ciencias y la tecnología, entre otras.

Lo anterior implica que existe un gran potencial para incrementar el ahorro interno y el crecimiento económico que puede ser aprovechado en la medida en que mayor número de mujeres opten por cursar estudios en las áreas "típicas" de los hombres, de manera que el ratio de ingresos femenino a masculino no tienda a caer. En otras palabras, los avances en el combate a la segregación sectorial de carreras universitarias pueden conducir a incrementar las tasas de ahorro interno y de crecimiento económico.

\section{Desigualdad de género, tributación y vulnerabilidad macroeco- nómica}

El gráfico 4 describe el papel de la reducción de la desigualdad de género y del aumento de la tributación en el aumento del ahorro y en la disminución de la vulnerabilidad macroeconómica de un país dado. 


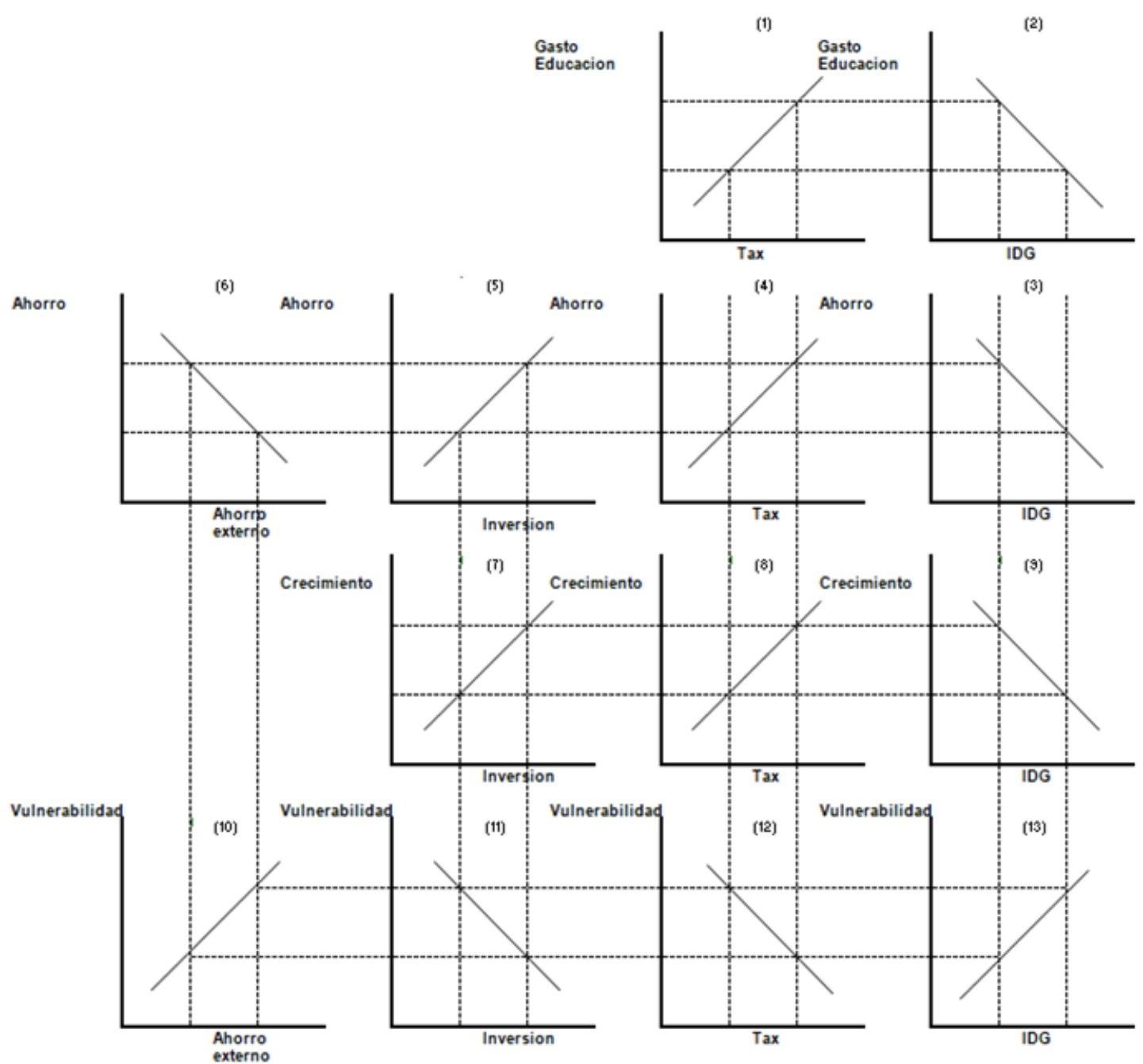

Gráfico 4. Desigualdad de género, tributación, ahorro, y vulnerabilidad macroeconómica.

Fuente: elaboración del autor.

El cuadrante 1 muestra el aumento del gasto público en educación a medida que aumentan los ingresos tributarios como porcentaje del PIB, Tax; mientras que en el cuadrante 2 se aprecia que el IDG cae ante el aumento del gasto en educación, según la evidencia presentada por Cáceres (2018) para una muestra de 12 países de América Latina con datos de $2012^{5}$.

El cuadrante 3 muestra la relación inversa entre el IDG y el ahorro interno, como se puso en evidencia en la ecuación 1 del cuadro 1 . Con esa base se construye en el cuadrante 4 la relación positiva entre la recaudación de impuestos y la tasa de ahorro interno. De acuerdo con la iidentidad macroeconómica, el cuadrante 5 presenta la relación positiva entre el ahorro interno y la inversión, mientras que el cuadrante 6 muestra la relación negativa entre ahorro interno y externo.

El cuadrante 7 representa el aumento de la tasa de crecimiento económico en respuesta al aumento de la inversión, lo cual permite construir en el cuadrante 8 una relación positiva entre el aumento de la tributación

\footnotetext{
${ }^{5}$ La ecuación estimada para representar el índice de desigualdad de género dio los siguientes resultados: Desigualdad de género $=0.5775-0.0871$ Cualich -0.0331 Gasto en educación (8.20) (1.48) (2.24)

$\mathrm{R}$ cuadrado $=0.44$

En donde Cualich representa una variable cualitativa que toma el valor de 1 en el caso de Chile.
} 
y la tasa de crecimiento económico, y en el cuadrante 9, la relación negativa entre esta tasa y el IDG, relación que ha sido puesta en evidencia por varios autores para países en vías de desarrollo, según consta en la reseña de Klasen (2018).

En el cuadrante 10, se postula la existencia de una relación positiva entre la tasa de ahorro externo, es decir, el déficit en la cuenta corriente, y la vulnerabilidad económica. El déficit en la cuenta corriente conduce a la acumulación de deuda externa y esta es un determinante de crisis financieras y cambiarias (Frankel y Saravelos, 1994), por lo que se puede asociar el déficit en la cuenta corriente con la vulnerabilidad económica.

Con esa base, se construye en el cuadrante 11 una relación negativa entre la vulnerabilidad y la inversión, y en el cuadrante 12 otra relación que hace ver que la vulnerabilidad cae al aumentar la tributación, mientras que en el cuadrante 13 se aprecia que la vulnerabilidad aumenta con el incremento de la desigualdad de género.

Para validar lo expuesto en el gráfico 4, se presenta en el gráfico 5, tomado de Cáceres (2017) y construido con datos de panel de países latinoamericanos, la relación positiva entre la ratio de ingresos tributarios a PIB, Tax, y las reservas internacionales per cápita, ambos de 2013. Esta relación obedece al supuesto de que una mayor recaudación tributaria permite incrementar el gasto social, especialmente el de educación, lo que conduce a la reducción de la desigualdad de género y al aumento del ahorro interno, y así a la caída del ahorro externo y, por tanto, a una menor acumulación de deuda externa. La caída de las reservas internacionales ha sido identificada por Sachs, Tornell y Velasco (1996) como detonador de crisis de balanza de pagos, mientras que Kaminsky, Elizondo y Reinhart (1996) reportaron que la reducción de las reservas era un indicador temprano de crisis financieras.

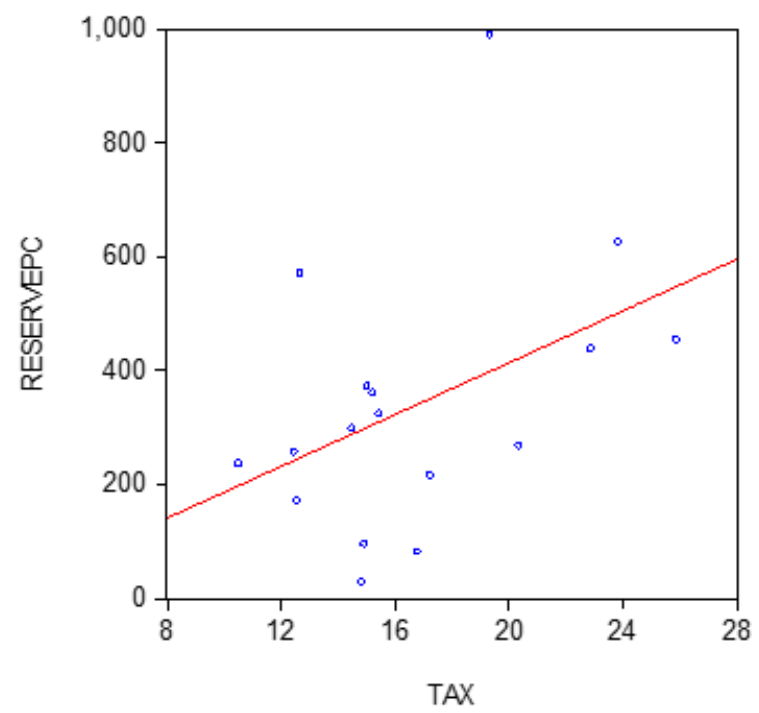

Gráfico 5. Tasa de tributación y reservas internacionales per cápita.

Fuente: elaboración del autor.

Se observa que en los países latinoamericanos a mayor esfuerzo tributario corresponde más baja vulnerabilidad económica ${ }^{6}$, que es la relación mostrada en el cuadrante 12 del grafico 4.

También se observa en la ecuación siguiente una relación negativa entre el IDG y las reservas per cápita, ambos de 2015, lo cual pone en evidencia el papel de la desigualdad de género en socavar la estabilidad macroeconómica y en aumentar la vulnerabilidad:

Reservas per cápita $=5926.747-11788.88$ IDG

\footnotetext{
${ }^{6}$ Se debe mencionar en este punto que no hay evidencia de que la tributación conduce al crecimiento del sector subterráneo de la economía; al contrario, la evidencia muestra que a mayor tributación corresponde más baja dimensión de la informalidad (véanse Dell'Anno, 2008; Sing, Jain-Chandra y Mohammad, 2012; y Friedman et al., 2000).
} 
(7.24) (5.93)

$\mathrm{R}$ cuadrado $=0.45$

Estos resultados hacen ver que la reducción de la desigualdad de género, así como el aumento del esfuerzo tributario, constituye valiosas políticas de prudencia macroeconómica.

\section{Genero y violencia.}

Es válido argumentar que la desigualdad de género representa una serie de situaciones en las cuales prevalecen acciones de discriminación contra la mujer en el mercado laboral, así como arbitrariedades y situaciones de violencia, acoso e intimidación, las cuales pueden pasar inadvertidas o no ser penalizadas. Esto puede dar lugar a la aceptación social de un patrón de conducta, sobre todo por la impunidad con que es recibido. Dicho patrón podría enviar mensajes a la sociedad de que la arbitrariedad es permitida y que puede expandirse a otras esferas de la sociedad. Es decir, la desigualdad de género puede dar lugar a un "efecto de dominó" de irrespeto a las leyes que conduce a la debilidad institucional, así como a un ambiente propicio a la violencia. O sea que la desigualdad de género podría "contaminar" el ambiente institucional de un país y pudiera servir como una "invitación" para irrespetar la ley.

Para corroborar el papel adverso de la desigualdad de género sobre el respeto a la ley, el cuadro 3 presenta una serie de ecuaciones econométricas entre indicadores de desigualdad de género y de violencia y de solidez institucional.

Cuadro 3. Desigualdad de género y violencia.

\begin{tabular}{|c|c|c|c|c|c|}
\hline & \multicolumn{5}{|c|}{ Ecuacion numero: } \\
\hline & (1) & $(2)$ & $(3)$ & (4) & $(5)$ \\
\hline & \multicolumn{5}{|c|}{ Variables dependientes } \\
\hline Variables & Tasa de & Tasa de & Control & Estado de & Ahorro \\
\hline independientes & Homicidios & Homicidios & Corrupcion & derecho & Interno \\
\hline \multirow{2}{*}{ Constante } & 27.6717 & 66.7069 & 3.2022 & 2.4733 & 19.2896 \\
\hline & $(2.51)$ & $(3.86)$ & $(4.24)$ & $(4.27)$ & $(11.97)$ \\
\hline Cuali & 65.4826 & 62.2848 & & & \\
\hline homicidios & $(9.04)$ & $(6.29)$ & & & \\
\hline \multirow{2}{*}{ Cualies } & & & & & -19.5891 \\
\hline & & & & & $(3.63)$ \\
\hline \multirow{2}{*}{ IDG } & 116.3109 & & -8.7338 & -7.4151 & \\
\hline & $(3.96)$ & & $(4.71)$ & $(5.28)$ & \\
\hline Empleo femenino / & -74.9182 & & & & \\
\hline masculino & $(2.78)$ & & & & \\
\hline \multirow{2}{*}{ Estado de derecho } & & & & & 3.7939 \\
\hline & & & & & $(1.80)$ \\
\hline $\mathrm{R} 2$ & 0.74 & 0.78 & 0.6 & 0.47 & 0.55 \\
\hline
\end{tabular}

La ecuación (1) del cuadro 3 indica que el IDG tiene un coeficiente significativo y positivo, implicando que a medida de que este índice aumenta, el número de homicidios anuales por 100,000 personas en el respectivo país también aumenta ${ }^{7}$. Esta ecuación explica casi tres cuartas partes de la variabilidad de la tasa de homicidios, lo que hace ver el papel determinante de la desigualdad de género en la violencia.

\footnotetext{
${ }^{7}$ La variable cualitativa cualihomicidos representa el caso de Honduras.
} 
La ecuación (2) implica que la tasa de homicidios cae a medida de que aumenta la ratio entre el empleo femenino al masculino. Esta ecuación explica cerca de 4 quintas partes de la varianza de la tasa de homicidios. Es decir, el empleo femenino es un verdadero antídoto para los homicidios (véase gráfico 6).

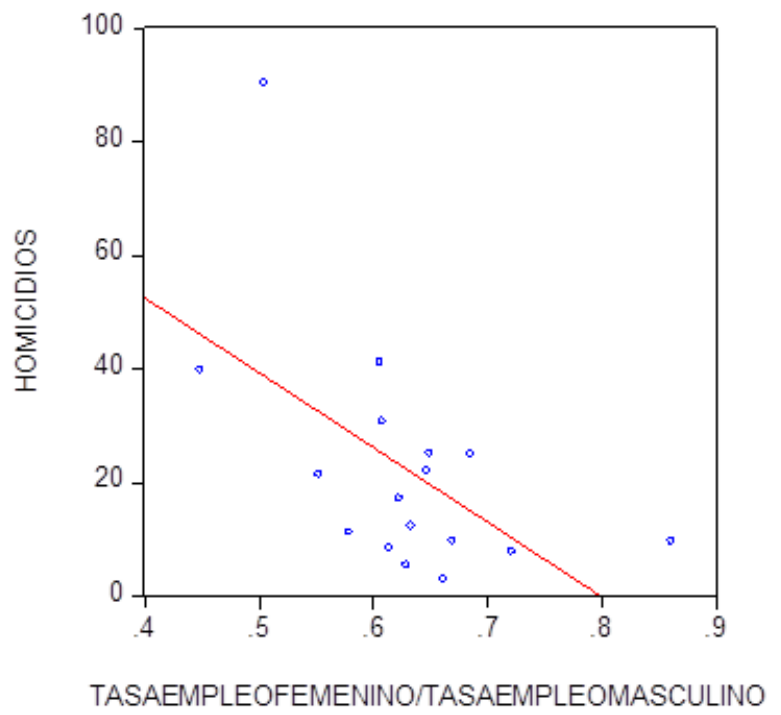

Gráfico 6. Ratio empleo per cápita anual femenino a masculino y tasa de homicidios.

Fuente: elaboración del autor.

El índice de control de corrupción, tomado del Banco Mundial ${ }^{8}$, es la variable dependiente en la ecuación (3) y el IDG tiene un coeficiente negativo y significativo, lo que denota su papel en socavar la solidez institucional.

En la ecuación (4), la variable dependiente es el índice de estado de derecho y muestra que el IDG tiene un coeficiente significativo y negativo, lo cual implica que, ante un aumento de la desigualdad de género, el marco institucional del respectivo país se deteriora.

El punto que se debe destacar es que la "contaminación" producida por la desigualdad de género es tal que la resultante debilidad institucional merma la capacidad de movilización de ahorro interno, como se aprecia en la ecuación (5) del cuadro 3. La ecuación indica que si Honduras mejorara su índice de estado de derecho de su valor de -0.83 al valor correspondiente a Chile (1.21), el incremento del índice de estado de derecho en 1.38 daría lugar al aumento de su tasa de ahorro interno en aproximadamente 5 puntos porcentuales.

En el contexto de los resultados anteriores, se debe hacer referencia a Wyndow, Li y Mattes (2013), quienes mostraron que la democratización de un país avanza en paralelo a la educación y participación laboral de las mujeres.

Hay que hacer referencia al estudio de Aizer (2007) que analizó el origen de la violencia contra la mujer en el hogar, cuyos resultados pusieron en evidencia que a medida de que la contribución monetaria de la mujer al hogar aumentaba, su poder de negociación mejoraba, ya que tenía opciones para salir de la relación, a la vez que su pareja temía perder el ingreso que la mujer aportaba al hogar; en consecuencia, la violencia familiar disminuía. Es decir, la mejor situación de la mujer en el mercado laboral le permitía "comprar seguridad" en la relación. La autora señaló que, si existiera la paridad de género en los salarios, la violencia doméstica disminuiría en $16 \%$ en el estado de California de los EE. UU.

Para investigar si estos resultados se reproducen en América Latina, se usaron datos de 7 países para los cuales existen datos anuales promedio del periodo 2005-2015 de incidentes de violencia sufrida por la mujer de parte de su esposo o compañero de vida, que se muestran en el gráfico 7 en términos de la razón del

\footnotetext{
${ }^{8}$ Worldbank governance indicators.org.
} 
ingreso anual per cápita femenino a masculino ${ }^{9}$. Este gráfico muestra lo contrario al modelo de Aizer, ya que la violencia contra la mujer en el hogar aumenta a medida de que los ingresos de la mujer mejoran con relación a los de su esposo o compañero de vida.

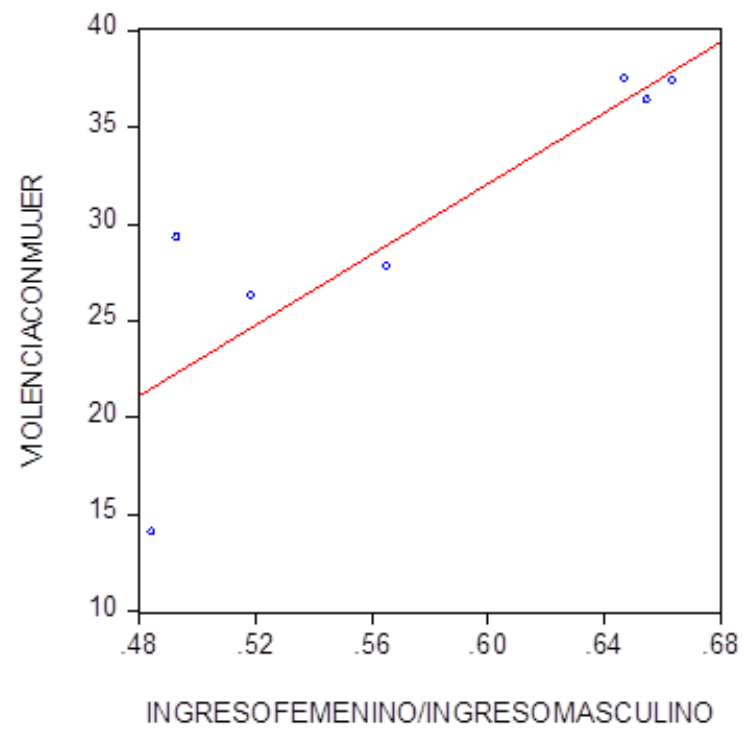

Gráfico 7. Ratio de ingreso anual per cápita femenino a masculino y casos anuales de violencia contra la mujer en el hogar.

Fuente: elaboración del autor.

La ecuación estimada es:

Actos de violencia contra la mujer

por su pareja $=-18.1952+86.1214$ Ingreso femenino/ingreso masculino

$(1.55)(4.35)$

$\mathrm{R}$ cuadrado $=0.72$

Es decir, el hecho de que las mujeres tengan crecientes ingresos relativos a los de sus parejas detona situaciones de agresión de sus esposos o compañeros de vida. Una extensa literatura ha mostrado que el aumento el ingreso de las mujeres hace que su pareja sienta que su masculinidad ha mermado, y teme perder su control del hogar, ante lo cual recurre a la violencia para afirmar su dominación (Molm, 1989; Johnson, 1995; Macmillan y Gartner, 1999).

Hay que apuntar que la violencia contra las mujeres no tiene relación con su educación, tanto las mujeres con altos y bajos niveles de educación son victimas de este flagelo. Mas bien, este es un problema de los hombres, que sienten que deben hacer palpable su dominio por medio de la violencia.

Un elemento que influye en estos resultados es la suposición por el hombre de que su compañera no va a recurrir a buscar el divorcio, y que no lo va a abandonar, es decir, asume que la mujer no tiene opciones, y por lo tanto el hombre puede dar rienda suelta a sus distorsiones mentales patriarcales consciente de que la esposa o compañera permanecerá en el hogar. Existe evidencia de los EE. UU. de que, con el propósito de que su cónyugue no abandone el hogar, y buscando mantener su posición dominante, el hombre recurre a oponerse a que su esposa opte por buscar empleo, y también a que continúe sus estudios, de manera que si esta terminara la relación tendría pocas oportunidades en el mercado laboral ${ }^{10}$. La ironía es que, al incrementar sus ingresos, la mujer contribuye a aumentar el ahorro interno y el crecimiento económico, y como "premio"

\footnotetext{
${ }^{9}$ Los países son México, El Salvador, Nicaragua, Perú, Colombia, Ecuador y Costa Rica.

${ }^{10}$ Véase una discusión de estos temas en Luis Rene Cáceres, "Mercado Laboral y Violencia Contra la Mujer", Contrapunto, San Salvador, 2012.
} 
recibe el abuso de su esposo o compañero de vida.

Esto señala la importancia de facilitar el proceso de divorcio, de eliminar la impunidad por actos de violencia en el hogar, y de ofrecer protección a las mujeres que se retiren de sus parejas violentas. Stevenson y Wolfers (2006) presentaron evidencia para los EE. UU. de que la puesta en vigencia de leyes que permitían el divorcio con base en la solicitud de solo un miembro de la pareja (en la mayoría de los casos, la mujer), ha dado lugar a la reducción de la violencia doméstica (homicidios). Estos autores también indican que el divorcio ha reducido el número de mujeres que se suicidan.

La importancia de medidas de este tipo se hace evidente al tomar en cuenta que los hijos que observan violencia doméstica reproducen el mismo patrón de violencia en su hogar cuando contraen matrimonio, es decir, la violencia contra la mujer en el hogar se "hereda" del padre, de una generación a otra (Pollak, 2002).

\section{Estrategia de país en materia de género}

Con el propósito de situar las acciones dirigidas a promover la igualdad de género en un marco estratégico que imparta directrices claras sobre el quehacer gubernamental en materia de género en el respectivo país, es conveniente estructurar una visión consolidada de lo que el gobierno plantea llevar a cabo en cuanto a la reducción de las disparidades de género en el corto y mediano plazo. Esta visión se enmarcaría en una estrategia de género de país.

Esta estrategia de país de género tendría los siguientes objetivos:

- Presentar una visión consolidada de los objetivos, los mecanismos, las acciones prioritarias y los indicadores de avance que permita mantener claridad y compromiso institucional en cuanto a las acciones que serán desarrolladas.

- Enriquecer los ejercicios de diálogo en el país respectivo, con miras a que las autoridades económicas nacionales, así como los sectores privados y la sociedad civil, se compenetren con la situación de las mujeres prevaleciente en el país, con las opciones de intervención y con los resultados esperados.

El esquema de la estrategia de país de género se presenta en el gráfico 8.

I) El primer elemento es el diagnóstico de la situación de la mujer en el país respectivo, que comprendería, entre otros, los siguientes aspectos:

- Legislación nacional en cuanto a herencia, propiedad de la tierra, acceso al crédito, aborto, divorcio, acoso en el trabajo, violencia intrafamiliar, violencia contra la niñez, salud reproductiva, vacaciones por maternidad y paternidad. Vacíos y obstáculos encontrados.

- Acceso de la mujer a actividades de emprendedurismo, especialmente relacionadas con acceso a crédito, apoyo institucional, etc.

- Actividades que están siendo desarrolladas en el área de género por organismos internacionales de desarrollo y por organizaciones nacionales.

- Políticas y programas que el gobierno está llevando a cabo en relación con la reducción de las brechas de género, recursos presupuestarios, estructura institucional de apoyo a la mujer.

- Esquema institucional nacional existente para la promoción de la igualdad de género.

- Lecciones aprendidas de las intervenciones del gobierno y de donantes en el país.

II) El siguiente elemento son los retos para el gobierno respectivo en cuanto a su acción en el apoyo a la paridad de género. Esto representa una declaración de las áreas prioritarias de acción y las razones para su selección. 
III) El siguiente elemento es el diseño de las estrategias a ser desarrolladas para el logro del avance en cada área prioritaria de atención. Esto comprende el establecimiento de los objetivos, las metas y los mecanismos a ser empleados.

Entre los mecanismos a que los gobiernos pueden recurrir para avanzar en el logro de los objetivos y las metas de la estrategia de genero se encuentran los siguientes: la pre-inversión, la identificación y preparación de proyectos, el apoyo a las instituciones nacionales que laboran en la promoción de la paridad de género, las acciones conjuntas con agencia internacionales de desarrollo, así como con ONG nacionales o internacionales, el diálogo con distintos grupos del país, estudios nacionales y regionales, etc.

IV) Otro elemento es la selección de indicadores de seguimiento y evaluación a ser empleados para la medición de los resultados. Un ejemplo de un juego de indicadores de seguimiento se presenta en el cuadro 4.

v) Se llevarían a cabo evaluaciones periódicas de la estrategia de país en materia de género para constatar el avance en los indicadores de progreso, así como para incorporar disposiciones recientes del gobierno respectivo en relación con políticas y programas relacionadas con género.

Las evaluaciones servirían de base para cambiar el énfasis en determinadas áreas o para incrementarlo en otras, de manera que la acción institucional mantenga siempre oportunidad y relevancia.

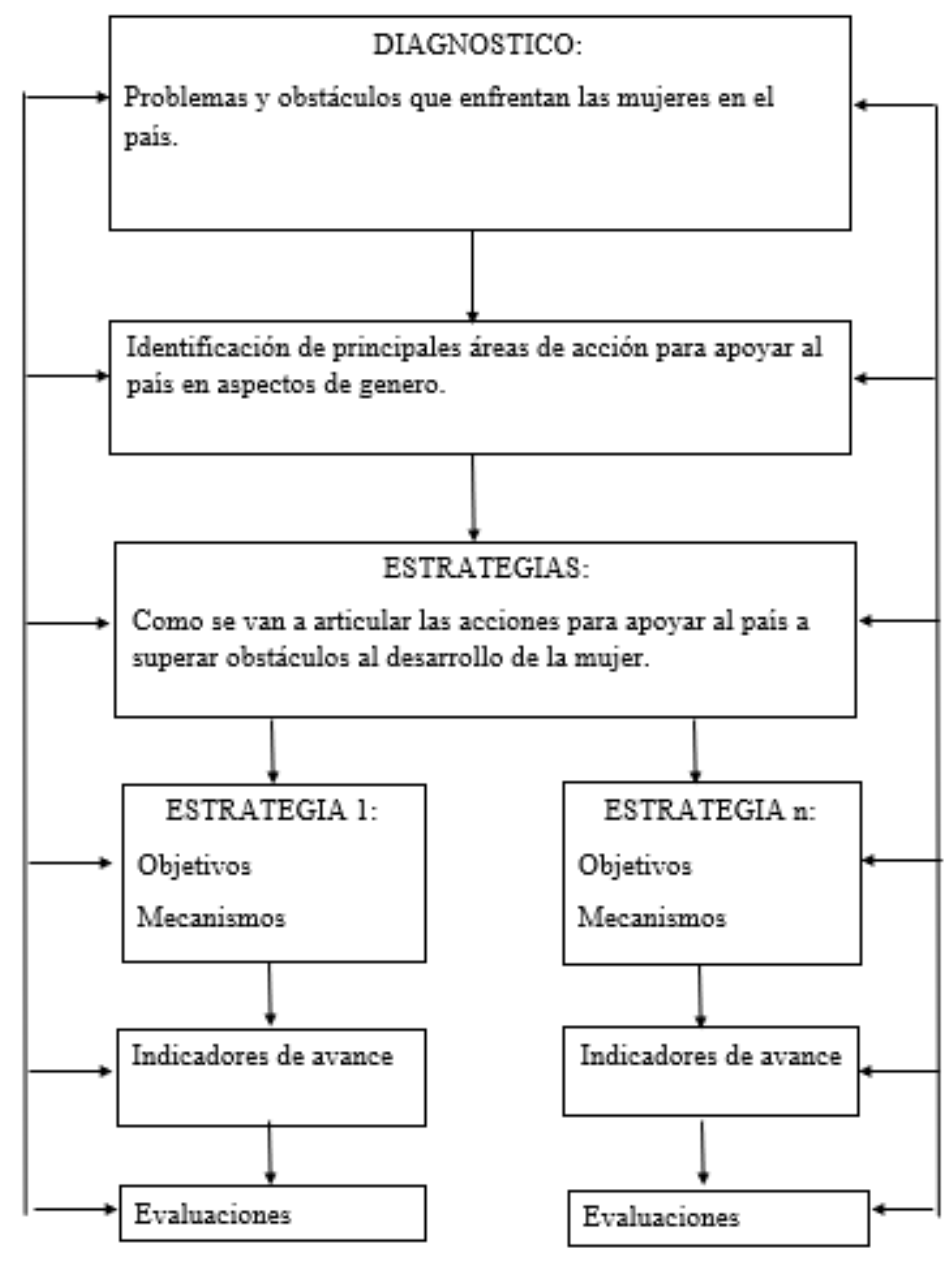

Gráfico 8. Esquema de estrategia de género de país.

Fuente: elaboración del autor. 
Cuadro 4. Indicadores de seguimiento de la estrategia de país de género.

\begin{tabular}{|l|l|l|}
\hline \multicolumn{2}{|l|}{ Indicadores del mercado laboral: } & Indicadores del sector educación: \\
\hline & Empleo femenino/masculino & Tasa matricula femenina/masculina en nivel: \\
\hline & Participación femenina/masculina & Educación preescolar \\
\hline & Informalidad femenina/masculina & Primaria \\
\hline & Desempleo juvenil femenino & Secundaria \\
\hline & Desempleo juvenil masculino & Terciaria \\
\hline & Acceso a servicios de salud ocupacional & Tasa de deserción escolar femenina/masculina \\
\hline & Discriminación de mujeres en el trabajo & Estudiantes universitarias que cursan carreras en: \\
\hline Indicadores del sector salud: & Ingeniería \\
\hline & Mortalidad Infantil & Ciencias \\
\hline & Mortalidad materna & Tecnología \\
\hline & Esperanza de vida al nacer & Puntajes en pruebas estandarizadas: \\
\hline & Acceso a servicios de salud mental & Femenino \\
\hline & Cobertura de vacunas & Masculino \\
\hline & Educación sexual & Urbano \\
\hline & Cobertura cuidado prenatal & Rural \\
\hline & Embarazos de adolescentes & Gasto público en educación. \\
\hline Indicadores de violencia & Indicadores de empoderamiento: \\
\hline & Numero de feminicidios & Número de mujeres diputados en el Congreso \\
\hline & Acoso en el trabajo & $\begin{array}{l}\text { Numero mujeres en el Ejecutivo como } \\
\text { Ministros, Vice Ministro. }\end{array}$ \\
\hline & Casos de "bullying" & Número de mujeres Alcaldes \\
\hline & Casos de violencia en el hogar & Número de mujeres Ejecutivas de grandes empresas \\
\hline
\end{tabular}

Fuente: elaboración del autor.

\section{Fondo Regional de la Mujer}

De especial importancia es la creación del Fondo Latinoamericano de la Mujer como un instrumento para facilitar la captación de recursos financieros y de cooperación técnica para canalizarlos a los países de acuerdo con un marco de políticas y programación definidas. Este mecanismo contribuiría a posicionar las tareas en beneficio de la paridad de género en un contexto más visible, a la vez que contribuiría a ahondar y a expandir el diálogo nacional y regional sobre la importancia del tema de género en los países miembros y sobre las falencias existentes.

El Fondo podría constituirse dentro de la estructura financiera, operativa y administrativa institucional de un organismo financiero regional existente en la región, con apoyo de capital inicial originado en aportes de países miembros, y de otros países u organismos internacionales, que estuviesen interesados en apoyar a las tareas de reducir la disparidad de género en la región. Las capitalizaciones se llevarían a cabo cada 5 años en el marco de objetivos y metas establecidos para ese periodo, que por supuesto serían congruentes con las estrategias de asistencia al país y de género de país.

El capital inicial sería complementado con aportes obtenidos que la institución obtendría de sus gestiones de consecución de recursos ante los mercados de capital y otros organismos financieros internacionales.

Las capitalizaciones subsecuentes se llevarían a cabo con base en documentos de reposición de recursos, que establecerían los correspondientes objetivos y metas y los montos de recursos a ser otorgados a los países en el periodo de 5 años. 
El Fondo podrá otorgar préstamos o cooperación técnica individualmente o en cofinanciamiento con otros organismos financieros internacionales. Los recursos de cooperación técnica se originarían en donaciones recibidas de países amigos o de organismos internacionales, así como en un porcentaje de las utilidades de la institución en un año, o periodo, dado.

El Fondo operaría en el marco y en las directrices de las políticas institucionales del organismo regional correspondiente, así como con base en sus propias políticas y reglamentos. Estos últimos comprenderían aspectos como los siguientes:

- Objetivos

- Recursos

- Programación operativa

- Estrategia de mediano plazo

- Evaluaciones

- Actividades a ser financiadas

- Recuperación de préstamos

- Elegibilidad de beneficiarios

- Inversiones de liquidez

- Supervisión

- Ingresos netos y su destino

- Contabilidad y auditoría

- Informes anuales a los Gobernadores.

\section{Conclusiones}

Los resultados presentados en este trabajo constituyen evidencia categórica de que la desigualdad de género representa un significativo obstáculo al aumento del ahorro interno y, por ende, del crecimiento económico. Además, el IDG tiene asociaciones negativas con los indicadores de control de corrupción y de estado de derecho, implicando que la desigualdad de género debilita las instituciones. De particular importancia es el papel adverso de la desigualdad de género sobre la estabilidad macroeconómica.

Estos resultados hacen ver la conveniencia de que la desigualdad de género se ubique en la agenda de desarrollo y estabilidad macroeconómica con la misma prioridad otorgada a la inflación, el tipo de cambio y las exportaciones. Esto es de especial importancia, ya que la efectividad de las políticas económicas tradicionales es afectada por la desigualdad de género. Es posible que una economía permanezca en una trampa de desigualdad de género, en la cual las políticas monetarias y fiscales tradicionales no hagan mella en la superación del estancamiento económico, en reducir la pobreza o en alcanzar la estabilidad financiera, en virtud de los impactos negativos sobre el ahorro interno, sobre la calidad de las instituciones, y sobre la estabilidad macroeconómica, ejercidos por la desigualdad de género, exhibiendo así la economía una tendencia a la crisis.

La serie de intentos fallidos para el logro del desarrollo económico, pasando de la sustitución de importaciones a la promoción de exportaciones, llegando al triste fiasco del Consenso de Washington, a la fiebre de 
acuerdos de libre comercio y al boom de los precios de bienes primarios de exportación, para desembocar en las recesiones ocasionadas por el fin de dicho boom, obligan a tomar en cuenta la evidencia presentada por Easterlin (1981) de que los países ahora desarrollados lograron grandes avances en su situación social, particularmente en la educación, como paso previo al despegue económico. También hay que tomar en cuenta el señalamiento de Stiglitz (2002) de que los países ahora desarrollados no pusieron en vigor, en sus pasos iniciales de industrialización, las medidas de políticas comprendidas en dicho Consenso, sino que se caracterizaron por el proteccionismo.

Es urgente enfilar los esfuerzos de desarrollo hacia el avance significativo en el desarrollo de capital humano y, en especial, en el logro de la paridad de género en sus diferentes manifestaciones. Estas son medidas que cuentan con respaldo económico e histórico, al contrario de la parafernalia de "libre comercio", "competencia", "mercados" y otros.

El principal obstáculo para incrementar la atención decidida del sector social ha sido la tradicional debilidad de las finanzas públicas, como resultado de la también tradicional oposición por los grupos de altos ingresos a incrementar la tributación, lo que ha significado un "tope" al monto de recursos destinados al gasto social. Esta situación es otra manifestación de la "cultura" de abuso, como la ha llamado la CEPAL (2018), que prevalece en la región y que socava los intentos hacia el desarrollo, dándole continuidad al subdesarrollo.

El deterioro que se observa en algunos países en materia de violencia y emigración irregular debería servir para concientizarse de que los modelos tradicionales no han procurado avances categóricos y autosostenidos en la ruta del desarrollo, y para emprender reformas fiscales conducentes a incrementar la tributación de los estratos de altos ingresos, destinando los recursos así obtenidos al desarrollo social. Hay que apuntar que el IDG explica tres cuartas partes de la tasa de homicidios de la región; dado que el costo de la violencia alcanza a nivel regional, según diversas estimaciones, entre 12 y 22 por ciento del PIB, las inversiones en la igualdad de género conducirían a reducir la violencia en un esquema en que dichas inversiones podrían pagarse por sí mismas.

Dada la evidencia de que la tasa de homicidios cae con el aumento de la ratio de empleo femenino a masculino, se deduce la importancia de otorgar la máxima prioridad al establecimiento de redes nacionales de guarderías infantiles que permitan a las madres continuar sus estudios e ingresar al mercado laboral, y a las madres jóvenes, especialmente a las solteras, continuar sus estudios, a la vez que se pueden complementar con programas de atención temprana a la infancia. Existe evidencia de que la incidencia de la pobreza es muy acentuada para las mujeres solteras jefes de hogar ${ }^{11}$.

Así mismo, en vista de la evidencia de los estudios que han mostrado que el aumento de los salarios conduce a incrementar la participación femenina (Smith y Ward, 1985; Tokman, 2006) con base en los resultados de este trabajo es válido argumentar que tal aumento contribuiría a incrementar el ahorro nacional y, por tanto, el crecimiento económico, y a reducir la violencia y a mejorar la calidad de las instituciones. Es decir, el aumento oportuno de los salarios es una inversión en paz, gobernabilidad y desarrollo.

Dada la evidencia de que la reducción de la desigualdad de género conduce a aumentar la tasa de ahorro interno, es válido postular que el incremento del ahorro contribuiría a aumentar la tasa de crecimiento económico del respectivo país y, por ende, sus importaciones de países vecinos aumentarían. Este desparrame de crecimiento económico entre países originado en la reducción de la desigualdad de género en uno o varios países, hace ver la conveniencia de que los países latinoamericanos adoptaran compromisos vinculantes de alcance regional para llevar a cabo políticas y programas destinados a reducir la desigualdad de género, y así aprovechar las que se podrían llamar economías externas de género. Esto se podría instrumentalizar por medio de la preparación de estrategias nacionales de género, preparadas a partir de la adopción por los países de directrices y compromisos regionales. Un complemento de dichas estrategias seria la creación del Fondo

\footnotetext{
${ }^{11}$ Véase Reporte de las Naciones Unidas, World's Women, 2015.
} 
Latinoamericano de la Mujer.

Los resultados obtenidos en este trabajo deberían ser otra motivación para colocar a la igualdad de género como un importante objetivo dentro de los programas de integración regional y subregional que se están dando en la región. Es decir, la dimensión de género puede otorgar a la integración regional un asidero importante que haga viable el logro de metas de desarrollo económico y social que han sido elusivas en el marco de los actuales esquemas de integración que descansan casi exclusivamente en el comercio de bienes. Los resultados también indican la importancia de que la reducción de la desigualdad de genero sea parte del conjunto de políticas de estabilización económica de los países.

\section{Referencias}

[1] AbuAl-Foul, Bassam (2010). "The Causal Relation Between Savings and Economic Growth: Some Evidence from MENA Countries", Topics in Middle Eastern and African Countries, 12, pp. 1-12.

[2] Acosta, Pablo (2006). "Labor Supply, School Attendance, and Remittances from International Migration: The Case of El Salvador", World Bank Working Paper no, 3909. Doi.org/10.1596/1813-9450-3903

[3] Agenor, Pierre Richard, Kamer Karakurum Ozdemir y Emmanuel Pinto Moreira, (2018). "Gender Gaps in the Labor Market an Economic Growth", Banco Mundial, documento de trabajo numero 8661, Washington DC, 2018. Doi.org/10.1596/1813-9450-8661

[4] Aizer, Anna (2007). "Wages Violence and Health in the Household", NBER working paper no. 13494. Doi: $10.3386 / \mathrm{w} 13494$

[5] Alguacil, Meite, Ana Cuadros y Vicente Orts (2004). "Does Saving Really Matter for Growth? Mexico (19702000), Journal of International Development, 61, pp. 281-290. Doi.org/10.1002/jid.1075

[6] Banda, Farida (2000). "Women, Human Rights and Development", en Realizing the Right to Development. Doi.org/10.1111/1467-7660.00167

[7] Buehn, Andreas y Friedrich Scheneider (2012). "Shadow Economies Around the World: Novel Insights, Accepted Knowledge, the New Estimates", International Tax and Public Finance, 19, pp. 139-171. Doi 10.1007/s10797011-9187-7

[8] Cáceres, Luis Rene (2017). "Stylized Facts about Happiness and Violence in Central America", Journal of Business Diversity, 17, pp. 38-78. Doi.org/10.33423/jbd.v17i1.1212

[9] CEPAL (2018). La Ineficiencia de la Desigualdad.

[10] Cerrutti, Marcela (2000). "Economic Reform, Structural Adjustment and Female Labor Force Participation in Buenos Aires, Argentina", World Development, 28. Doi: 10.1016/S0305-750X(99)00163-1

[11] Dell'Anno, Roberto y Helen Solomon (20008). "Shadow Economy and Unemployment Rate in the USA: Is There a Structural Relationship? An Empirical Analysis", Applied Economics, pp. 2537-2555. Doi.org/10.1080/00036840600970195

[12] Engerman, Stanley L. y Kenneth L. Sokoloff (2005). "Colonialism, Inequality, and Long-Run Paths of Development", NBER Working Paper no. 11057. Doi: 10.3386/w11057

[13] Esso, Loesse Jaques y Yaya Keho (2010). "The Savings-Investment Relationship: Cointegration and Causality Evidence from Uemoa Countries", International Journal of Economics and Finance, 2, pp. 174-180.

[14] Fernandes, Reynaldo y Fabiana de Felicio (2005). "The Entry of the Wife into the Labor Force in Response to the Husband's Unemployment: A Study of the Added Worker Effect in Brazilian Metropolitan Areas", Economic Development and Cultural Change, 53(4), pp. 887-911. Doi.org/10.1086/429357

[15] Floro, Maria Sagrario y Stephanie Seguino (2002), "Gender Effects on Aggregate Saving", World Bank, Gende and Development Policy Research Report numero 23.

[16] Friedman et al (2000). "Dodging the Grabbing hand: The Determination of Unofficial Activity". 
[17] Hanushek, Eric A. y Ludger Woessmann (2009). "Schooling, Cognitive Skills and the Latin American Growth Puzzle", NBER working paper 15066. Doi: 10.3386/w15066

[18] Hinz, Richard, David McCarthy, y John Turner (1996). "Are Women Conservative Investors?:Gender Differences in Participant-Directed Pension Investments,", en Michael Gordon, Olivia Mitchell, and Marc Twinney(eds.), Positioning Pensions for the Twenty-First Century, University of Pennsylvania Press, Philadelphia, PA, pp. $145-156$.

[19] Hungerford, Thomas (1999). "Saving For a Rainy Day: Does Pre-Retirement Access to Retirement Savings Increase Retirement Saving?," Mimeo, Social Security Administration.

[20] Jadotte, Evans (2009). "International Migration, Remittances and Labour Supply", Research Paper no. 2009/28, ONU-WIDER.

[21] Johnson, M. (1995). "Patriarchal Terrorism and Common Couple Violence: Two Forms of Violence Against Women", Journal of Marriage and the Family, 57, pp. 283-294.

[22] Frankel, Jeffrey y George Saravelos (2012). Can Leading Indicators Asses Country Vulnerability, Journal of International Economics, 87, num. 2, pp. 216-231.

[23] Karahan, Ozcan (2018). "The Causal Relation Between Savings and Economic Growth" in Turkey", in The Political Economy of Development in Southern Europe, edited by Spyros Roukaras, Persefoni Polydronidou,y Anastasios Karasavvoglu, Springer, New York.

[24] Klasen, S (2018). "The Impact of Gender Inequality on Economic Performance in Developing Countries", Annual Reviews of resource Economics, 10, pp. 279-298. Doi.org/10.1146/annurev-resource-100517-023429

[25] Kumar, Shubh (1978). "Role of the Household Economy in Child Nutrition at Low Incomes: A Case Study in Kerala," Occasional Paper No. 95, Department of Agricultural Economics, Cornell University, Ithaca, NY.

[26] Macmillan, Ross y Rosemary Gartner (1999). "When She Brings Home the Bacon: Labor-Force Participation and the Risk of Spousal Violence Against Women", Journal of Marriage and Family, 61(4), pp. 947-958.

[27] Markowitz, S. (2000). "The Price of Alcohol, Wife Abuse and Husband Abuse", Southern Economic Journal, 67, pp. 279-303.

[28] Mckenzie, David (2003). "How do Households Cope with Aggregate Shocks? Evidence from the Mexican Peso Crisis", World Development, 31, pp. 1179 - 1199.

[29] Misztal, Piotr (2011). "The Relationship Between Saving and Economic Growth in Countries with Different Levels of Development", The Central European Journal of Social Sciences and Humanities, 7, pp. 17-29.

[30] Molinas Vega, Jose R., Ricardo Paes de Barros, Jaime Saavedra, y Marcelo Giugale, con Louise Cord, Carola Pessino y Amer Hasan (2011). Do Our Children Have a Chance? Banco Mundial. Doi.org/10.1596/978-0-82138699-6

[31] Molm, Linda (1989). "Punishment Power: A Balancing Process in Power-Dependence Relations", American Journal of Sociology, 94. Doi.org/10.1086/229159

[32] Loayza, Norman (1997). "The Economics of the Informal Sector. A Simple Model and Some Empirical Evidence fron Latin America". World Bank Policy Research Working Paper, 1727. Doi.org/10.1596/1813-9450-1727

[33] Oladipo, Olajide S. (2010). "Does Saving Really Matter for Economic Growth in Developing Countries? The Case of a Small Open Economy", International Business and Economics Research Journal, 9. /doi.org/10.19030/iber.v9i4.556

[34] Parker, Susan y Emmanuel Skoufias (2004). "The Added Worker Effect Over the Business Cycle: Evidence from Urban Mexico", Applied Economics Letters, 11, pp. 625, 630. Doi.org/10.1080/1350485042000235693

[35] Patra, Suresh Kumar y Arfat Ahmad Sofi (2016). "Causal Nexus Between Growth and Savings in India Using Nonlinear Causality Approach", International Journal of Research Methodology in Social Science, 2, pp. 6-18.

[36] Paz, Jorge (2009). "El Efecto del Trabajador Adicional: Evidencia para Argentina (2003-2007)", Cuadernos de Economía, 46, pp. 225-241. /dx.doi.org/10.4067/S0717-68212009000200005 
[37] Phillips, Peter C. B. y Bruce E. Hansen (1990). "Statistical Inference in Instrumental Variables Regressions with I(1) Processes", Review of Economic Studies, 57. "Statistical Inference in Instrumental Variables Regressions with I(1) Processes", doi.org/10.2307/2297545

[38] Pollak, Robert A. (2002). "An Intergenerational Model of Domestic Violence", NBER working paper no. 9099. Doi.org/10.1007/s00148-003-0177-7

[39] Ramesh, Jangili (2011). "Causal Relationship Between Saving, Investment abnd and Economic Growth for India. What Does the Relation Imply?", document de trabajo, MPRA.

[40] Rodriguez, Edgard R. y Erwin R. Tiongson (2001). "Temporary Migration Overseas and Household Labor Supply: Evidence from Urban Philippines", International Migration Review, 35, pp. 709-725. Doi.org/10.1111/j.17477379.2001.tb00037.x

[41] Sachs, Jeffrey, Aaron Tornell y Andres Velasco (1996). "Financial Crisis in Emerging Markets: The Lessons from the 1995", Brookings Papers on Economic Activity, 26, pp. 147-225. Doi: 10.3386/w5576

[42] Sahay, Ratna, y Martin Cihak (2018), "Women in Finance. A Case for Closing the Gaps, IMF Staff Discussion Note 18/05, International Monetary Fund.

[43] Seguino, S. (2000). "Gender Inequality and Income Growth: A Cross-country Analysis", World Development, 28, pp. 1211-1230.

[44] Seguino, S., y M. S. Floro (2003). "Does Gender have any Effect on Aggregate Saving?", International Review of Applied Economics, 17, 2003, pp. 147-166. Doi.org/10.1080/0269217032000064026

[45] Singh, Anoop, Sonali Jain-Chandra y Adil Mohommad (2012). "Inclusive Growth, Institutions, and the Underground Economy", IMF working paper 12/47.

[46] Skoufias, Emmanuel y Susan Parker (2006). "Job Loss and Family Adjustments in Work and Schooling During the Mexican Peso Crisis", Journal of Population Economics, 19, pp. 163-181.

[47] Smith, James y Michael Ward (1985). "Time Series Growth in the Female Labor Force", Journal of Labor Economics, 3, pp. 59-90. Doi.org/10.1086/298076

[48] Stevenson, B. y J. Wolfers (2006). "Bargaining in the Shadow of the Law: Divorce Laws and Family Distress", Quarterly Journal of Economics, 121, pp. 267-288. doi.org/10.1093/qje/121.1.267

[49] Swamy, et al (2001). "Gender and Corruption", Journal of Development Economics, 64, pp. 25-55. 10.1016/S0304$3878(00) 00123-1$

[50] TERCE, Tercer Estudio Regional Comparativo y Explicativo (2016). Unesco, Santiago de Chile, 2016.

[51] Thomas, Duncan (1992). "The Distribution of Income and Expenditure Whithin the Household", IFPRI World Bank Conference on Intrahousehold Resource Allocation: Policy Issues and Research Methods, Washington D.C.

[52] Tokman, Victor (2000). "El Empleo y la Crisis: Efectos y Politicas", CEPAL, Serie Macroeconomia del Desarrollo, 100.

[53] Van Ewijk, C., D. Draper, H. terRele, y E. Westerhout (2006). "Ageing and the Sustainability of Dutch Public Finances", CPB Netherlands Bureau for Economic Policy Analysis.

[54] Velutin, Guillermo (2008). "Measuring the Informal Economy in Latin America and the Caribbean", IMF working paper 08/102.

[55] Wyndow, Paula, Jianghong Li y Eugen Mattes (2013). "Female Empowerment as a Core Driver of Democratic Development: A Dynamic Panel Model from 1980 to 2005", World Development, 32, pp. 34-54. 